\title{
Input Preshaping Control of the safe arm with MR-based Passive Compliant Joints
}

\author{
Seung-kook Yun, Sungchul Kang, Munsang Kim \\ Intelligent Robotics Research Center \\ Korea Institute of Science and Technology \\ 39-1 Hawolgok-dong, Sungbuk-ku, Seoul, 136-791, Korea \\ \{arumi, kasch, munsang\}@kist.re.kr
}

\begin{abstract}
In this paper, design and control of a safe arm with passive compliant joints(PCJ) are presented. A new manipulator with passive compliance property has been developed at KIST. Strategy for the joint control method is implemented. Because the PCJ is a flexible joint, unwanted vibration is occurred during the joint motion. To reduce this vibration, we use the input preshaping method which is motivated by the input shaping technique(IST) based on impulse response. The control method for a time-varying system like the safe arm is described in detail. The result of simulations and experiments proves that a fast motion of the safe arm without residual vibration can be achieved.
\end{abstract}

Index Terms - Passive Compliant Joint, Magneto-rheological Damper, Input Preshaping, Input shapint Technique

\section{INTRODUCTION}

Robots are recently expected to provide various kind of services directly to human in human-robot coexisting environment. Considering physical human-robot interaction, safety is one of the most important issues to be accomplished. To make a compliant robot arm can be a good way to greatly enhance the safety performance. Generally there are two strategies to realize the robot compliance: active and passive. Active compliance approach usually makes use of the feedback signals from force/torque sensors equipped either on a robot hand or at joints so that the robot controller may detect the applied external force and generate a proper response. The active compliance approach may have a limit in delayed control and unreliable safety in case of electrical malfunctioning, even though it can offer high program ability for compliance control. In this case, a manipulator with active compliance function may cause either damage to human or failure of power transmission due to the shock when unexpected contact occurs. On the other hand, passive compliance can be realized by applying a passive mechanism to produce an appropriate reaction to the applied forces. Focusing on reliable and safe interaction, therefore, this work is a challenge in the passive approach. Especially, the design of the safe arm with a magneto-rheological rotary damper and rotary springs is newly proposed. With this MR damper and rotary springs, a passive compliance joint(PCJ) is developed for the safe arm of a service robot[1][2].

The safe arm has advantage in simple compliance control when compared with the arm with active compliance control. When controlling the joint with a PCJ, any additional programming for the control motion program is not needed

\author{
Seong-Sik Yoon \\ Intelligent Robotics Laboratory \\ Toyota Technological Institute \\ 2-12-1, Hisakata, Tempaku-ku, Nagoya 468-8511, Japan \\ rooook@toyota-ti.ac.jp
}

since the PCJ is inherently passive mechanism. Unless the spring part or the MR damper part is broken mechanically, we can guarantee that compliance control works even if no power is supplied to the electric part of the arm. As well, the compliance control works regardless of the contact point on the arm surface while the arm with active compliance control which uses force/torque sensor at its wrist cannot detect the external force or torque at the contact point of lower links.

In human-robot interaction environment, the safe arm has several merits as previously commented. But unwanted vibration may occur because of springs included in each PCJ during the fast motion of each joint. Although the damper of the PCJ may partly suppresses vibration, a fast motion cannot be performed without vibration. This vibration may cause an unstable control or unwanted collision to the environment which degrades safety performance..

The Input Shaping Technique(IST)[3] based on impulse responses has been considered as a good way to solve problem of vibration because of its simple structure and high efficiency.. Although the IST can be an excellent solution to reduce vibration for a linear time invariant system, it seems not much effective for a non-linear or a time varying system. Since the safe arm is a time varying system in which its inertia property changes by the arm configuration, a IST for a time varying system is needed to reduce vibration of the arm.

A thesis that a proper IST filter with two impulses can eliminate a residual vibration of a time invariant system is proved by Park[4]. However it is almost impossible to identify the exact time for the second impulse. There have been a couple of studies to apply the IST to a time varying system. Rappole[5] applied a time varying input shaping technique(TVIST) to a two link flexible manipulator, Park[6] proposed a practical method of TVIST to reduce vibration of an industrial robot. The former used a look-up table which contained information on configuration-dependent frequency, while the latter used a simple equation relating a period of vibration to a length which is from the swing axis to the wrist axis along the horizontal line. Although these two methods can be practical way to reduce vibration of a time varying system, an estimation of timing of the second impulse time is always incorrect because of dynamics of the system.

In this paper, we propose an effective method for the vibration control of the safe arm with a properly preshaped trajectory. Although the preshaping method is motivated by the IST based on impulse responses, it has slightly different 
structure to reduce vibration both at the start and the end position.

This paper is organized as follows. Section 2 introduces the design of the PCJ and the safe arm. Section 3 describes the input preshaping method for the manipulator with PCJs. Section 4 shows some simulation and experiment results. In Section 5, a conclusion and further works are stated

\section{SAFE ARM DESIGN}

\section{A. Design of passive compliant joint(PCJ)}

The design of the passive compliant joint[1][2] is shown in Fig. 1. The PCJ is composed of a magneto-rheological (MR) rotary damper and rotary springs. The rotary springs become a source of vibration while they give a PCJ's compliant property. Therefore a viscous damper is implemented using the developed MR damper which has a maximum $21 \mathrm{Nm}$ force output. The PCJ has a resolver sensor of 16-bits high resolution to read the relative position between the reducer(harmonic drive gear) and the link caused by the spring displacement. The relative position is converted into a relative velocity signal through numerical differentiation and sent to a damping tuner which converts coulomb friction property of the MR damper into viscous one. Note that the damper and spring are located in parallel between the reducer and link. A driving unit is made up of a DC motor, an encoder, a timing belt, and a harmonic drive gear.

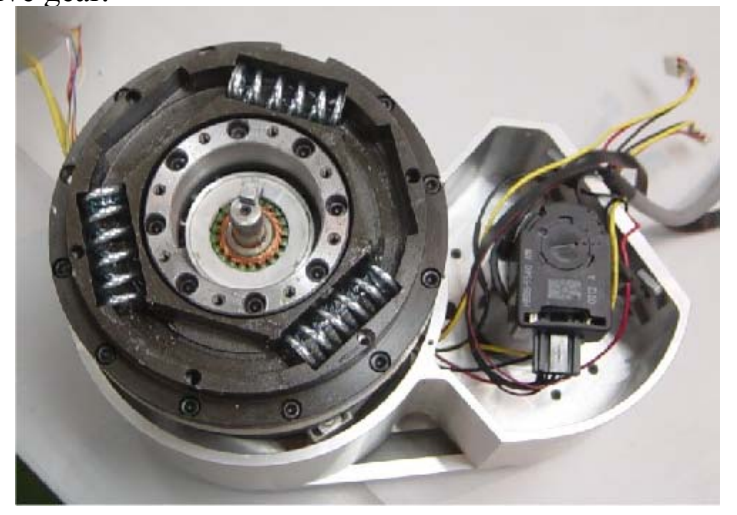

Fig.1 A complete set of the PCJ

\section{B. Safe Arm Design}

A safe arm including the MR-based passive compliant joints is designed for service robot applications. First three joints from the arm base includes the PCJ each of which has different capacity of compliance. The safe arm has additional passive mechanism with the visco-elastic material as shown in Fig. 2.

When collision between a robot and human occurs, soft covering of the robot arm is necessary to attenuate the impact force, that is, the force at the start time of collision; Even in case of the safe arm, the impact force can not be absorbed effectively by only PCJ mechanism since the arm has its inertia. PORON SR-S-24(urethan foam) is used for the soft covering of the safe arm. The thickness of the cover is set to $5 \mathrm{~mm}$ taking account of the size of the arm. The specification of each PCJ is shown in Table I.

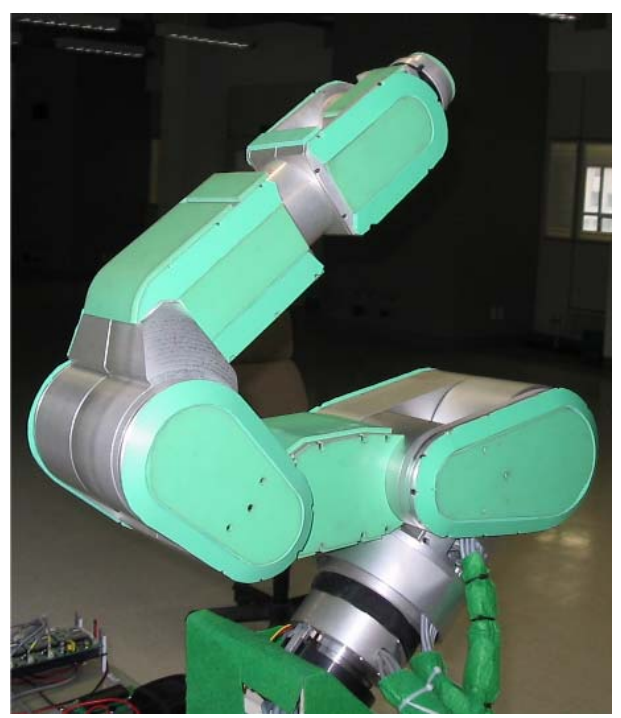

Fig. 2 KIST safe arm

TABLE I

SPEC OF EACH PCJ

\begin{tabular}{|l|c|c|c|}
\hline \multirow{2}{*}{} & \multicolumn{3}{|c|}{ Axis number } \\
\cline { 2 - 4 } $\begin{array}{l}\text { Spring Constant } \\
\text { (Nm/rad) }\end{array}$ & 350 & 420 & 210 \\
\hline $\begin{array}{l}\text { Maximum dampingTorque } \\
\text { (Nm) } \\
\text { Viscous damping coefficient } \\
\text { (Nm sec/rad) }\end{array}$ & 17.3 & 20.5 & 6.97 \\
\hline
\end{tabular}

Fig. 3 schematic drawing of a PCJ

\section{INPUT PRESHAPING METHOD FOR THE SAFE ARM}

Although the PCJ enables passive compliance for the safe arm, it also can be a source of unwanted vibration. This vibration may cause an unstable control or unwanted collision to the environment. Especially, a lot of residual vibration can occur during the fast rotation of the PCJ. In Fig.3, a schematic picture of a PCJ is shown. A spring and a damper make the PCJ into a flexible joint To cope with this problem, we need a vibration controller for the PCJ.

\section{A. Input Shaping Technique(IST)}

As an effective solution to reduce this vibration, an input shaping technique(IST) based on impulse response has been proposed[3]. The IST uses two impulses to get rid of residual 
vibration. The IST uses two impulses to get rid of residual vibration. Fig.4 shows how the IST works with two impulse responses. If the second impulse time is properly selected, the superposed response will have no vibration after the time of the second impulse. The IST can be considered as a simple filter since these two impulses are to be convoluted with a desired trajectory or an open loop control input. as shown in Fig.5.

$1^{\text {st }}$ impulse.

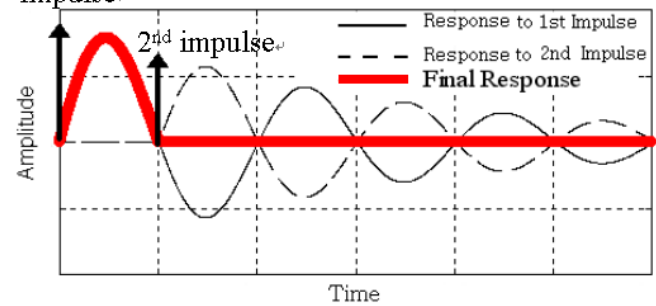

Fig. 4 Vibration suppression via IST

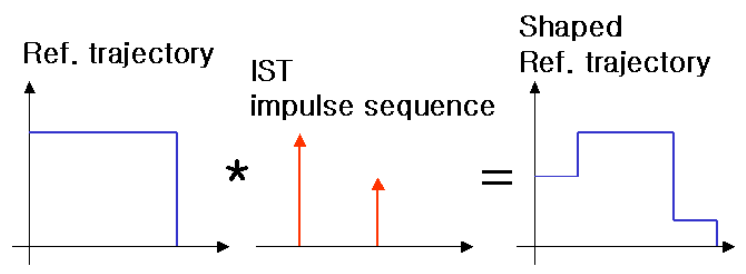

Fig. 5 Shaping reference trajectory using the IST

\section{B. Estimation of Time Varying Period}

The IST can be an excellent solution to reduce vibration for a linear time invariant system, but it is not so effective for a non-linear or a time varying system. Since the safe arm is a time varying system in which its property changes during the motion of the arm. Although it is almost impossible to find out the exact timing of the second impulse for a time varying system, there have been a couple of approaches to find the sequence for a time varying system For this time varying input shaping technique(TVIST), we need to know periods of vibration in all configurations that the arm can have in its workspace.

A dynamic equation of $i$-th PCJ of the safe arm excluding gravity is as follows:

$$
J_{i}\left(\theta_{i}\right) \ddot{\theta}_{i}+C\left(\theta_{i}, \dot{\theta}_{i}\right)+K_{i}\left(\theta_{i}-\theta_{m i}\right)=\tau_{i}
$$

where $J_{i}$ is a inertia term, $C$ is a coriolis and centrifugal term, $K_{i}$ is a spring constant, $\theta_{m i}$ is a motor angle of $i$-th axis and $\tau$ is a control torque.

A period of vibration for the $i$-th PCJ can be expressed as follows:

$$
T_{i}=2 \pi \sqrt{\frac{J_{i}}{K_{i}}} \quad(i=1,2,3)
$$

Because the safe arm is a serial manipulator, a period of vibration for each joint is function of next joint angle assuming a spring constant is time-invariant. We can consider the period of vibration for the $3 \mathrm{rd}$ joint as constant because change of inertia along the wrist configuration is comparably small.
Therefore, time varying ISTs for the 1 st and 2 nd joint is needed. In this research, vibration control of 2 nd joint is only implemented since the same method can be applied to 1 st joint control.

To determine the proper parameter for TVIST, the periods of vibration at different configurations are measured by experiments. In Fig.5, the period of vibration for the 2 nd joint according to change of the 3rd joint angle is shown. We can expect the period of vibration is proportional to square root of $\cos \theta_{3}$ because inertia is proportional to square root $\cos \theta_{3}$. The curve of period of vibration has some similarity to the curve as follows:

$$
T_{\text {period }}=\sqrt{\alpha+\beta \cos \left(\theta_{3}-\theta_{o}\right)}
$$

where

$$
\alpha=0.2, \beta=0.22, \theta_{\mathrm{o}}=30^{\circ} .
$$

We can make a table of the period of vibration along each joint configuration using obtained data. When controlling the PCJ, we can use the table or the equation.

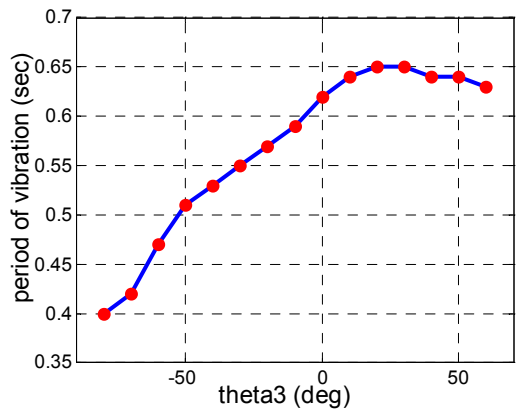

Fig. 6 Period of vibration at 2nd joint along joint configurations

\section{C. $\quad$ Modified TVIST}

In TVIST, a controller selects the second impulse timing of the IST impulse sequence by comparing the joint configuration with tablized measured parameters, that is, the periods of vibration. Even if the selection is correct and the measured data are exact ones, however, there is still some vibration because of timing error due to the dynamics of the system. When the inertia increases, the selected time becomes later than the correct timing, and vice versa as shown in Fig. 7.

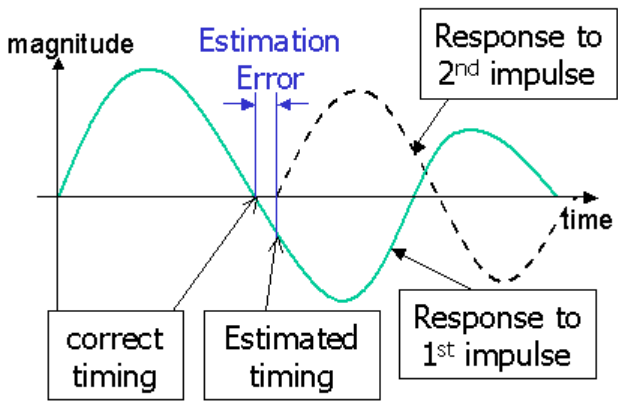

Fig. 7 Estimation error of the second impulse timing 
Fig.8 shows this error clearly. The picture shows the PCJ displacement defined as follows:

$$
\theta_{r}=\theta_{L}-\theta_{m}
$$

where $\theta_{L}$. and $\theta_{m}$ are a link angle and a motor angle shown in Fig.3. The simulation is performed with the 2nd PCJ system. In the simulation, inertia increases linearly as follows:

$$
\begin{array}{ll}
J=J_{o}+t & \text { for } t<1 \\
J=1+J_{o} & \text { otherwise }
\end{array}
$$

where $J_{o}=0.55 \mathrm{kgm}^{2}$, which is the estimated value of the minimum inertia for the $2^{\text {nd }}$ joint. Vibration frequency is changed from $1.65 \mathrm{~Hz}$ to $2.65 \mathrm{~Hz}$.

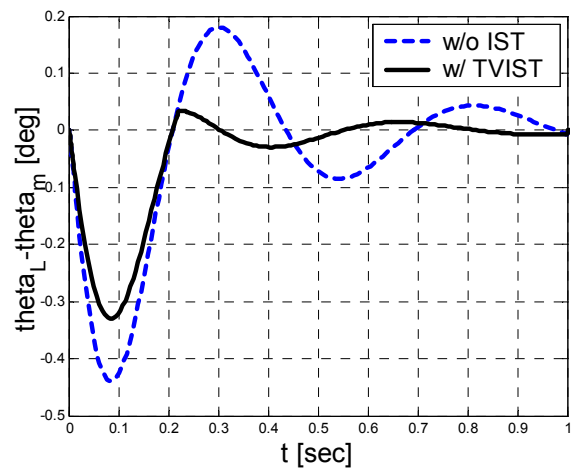

Fig. 8 Simulation : using TVIST

Although a lot of vibration is suppressed by TVIST, you can see that small vibration still remained even in the simulation.

To compensate this error caused by dynamics of the system, we set the second impulse time as a mean of the estimated time in TVIST. Then the second impulse time guessed at the motion-start as follows:

$$
T_{\text {half }}=\frac{T_{\text {half_start }}+T_{\text {half_TVIST }}}{2}
$$

where

$$
\begin{gathered}
T_{\text {half_start }}=\left.2 \pi \sqrt{\frac{J_{i}\left(\theta_{i}(t)\right)}{K_{i}}}\right|_{\theta_{i}=\theta_{\text {start }}(t=0)} \\
T_{\text {half_TVIST }}=\left.2 \pi \sqrt{\frac{J_{i}\left(\theta_{i}(t)\right)}{K_{i}}}\right|_{\theta_{i}=\theta(t=\text { estimated time })}
\end{gathered}
$$

Fig .9 shows the simulation results of the same system as in Fig.8. The response with modified TVIST has almost no vibration compared with other responses. For the safe arm, the response of the system is much faster than changing rate of inertia. Therefore, we can see this modified TVIST method works well for the safe arm.

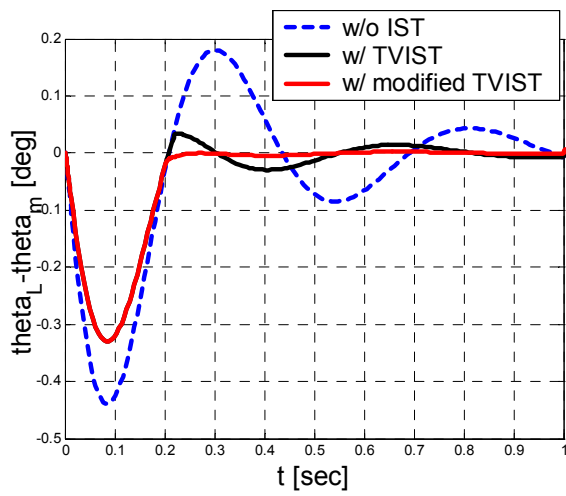

Fig. 9 Simulation: Using modified TVIST

\section{Final Input Preshaing Method}

Generally, there are two phases of vibration during motion of a flexible joint. The first one is excited by start motion, and the second is by end motion. In Fig.10, a simulation result is shown when the 2 nd axis moves by 30 degree during one second. Looking at the right part of the drawing carefully, we can find that there are two kind of vibration; the first one is started at zero second, and the second is started at one second which is the start time of steady state.

Of course, these two vibrations have different frequencies and periods since the safe arm is a time varying system. Therefore, we need two different IST filters to eliminate both of vibration. We designed a modified TVIST filter for the 1st vibration, and a conventional IST filter for the 2 nd vibration because the system is almost time invariant when the joint motion reaches the steady state.
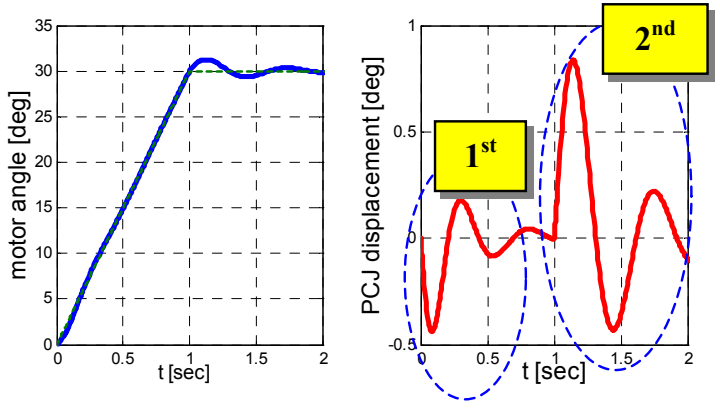

Fig. 10 two phase of vibration

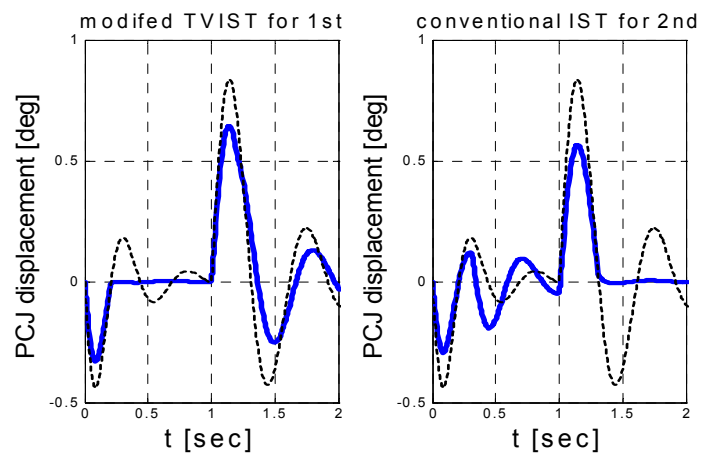

Fig. 11 Simulation: ISTs for two kinds of vibration 
In Fig.11, simulation results of each case is shown, the left part results from the controller with a modified TVIST, and the right with a conventional IST for the 2 nd vibration. We can see the modified TVIST can eliminate the 1st vibration but 2 nd vibration, and the conventional IST can eliminate only 2 nd vibration. In case of a LTI system, we can easily eliminate both of vibration by using a merged IST filter by convoluting the $1^{\text {st }}$ IST filter to 2nd one. However, this principle does not work in case of a LTV system.

Now we propose a new input-preshaping method for the safe arm, which is motivated by the IST. In previous simulations, we can get IST parameters of each vibration which are composed of each magnitude of two impulse and the second impulse timing. All parameters we need to know are listed as follows:

$A_{1}$ : magnitude of $1 \mathrm{st}$ impulse for the modified TVIST

$A_{2}$ : magnitude of 2 nd impulse for the modified TVIST

$T_{2 A}$ : half period of 1 st vibration

$B_{1}$ : magnitude of $1 \mathrm{st}$ impulse for the conventional IST

$B_{2}$ : magnitude of 2 nd impulse for the conventional IST

$T_{2 B}$ : half period of 2 nd vibration

Where

$$
\begin{aligned}
& A_{1}+A_{2}=1 \\
& B_{1}+B_{2}=1
\end{aligned}
$$

to guarantee the same steady-state value.

Using these parameters, we set a new trajectory as follows:

$$
\begin{array}{ll}
\theta_{d}^{*}(t)=R \cdot\left[A_{1} \theta_{d}(t)+A_{2} \theta_{d}\left(t-T_{2 A}\right) u\left(t-T_{2 A}\right)\right] & 0<t<T_{f}, \\
\theta_{d}^{*}(t)=R \cdot\left[B_{1} \theta_{d}(t)+B_{2} \theta_{d}\left(t-T_{2 B}\right) u\left(t-T_{2 B}\right)+\alpha\right] & T_{f}<t
\end{array}
$$

where $T_{f}$ is the time of steady state point,

$$
\begin{gathered}
R=\frac{\int_{0}^{T_{f}} \dot{\theta}_{d} d t}{\int_{0}^{T_{f}+T_{2 B}} \dot{\theta}_{d}^{*} d t}, \\
\alpha=A_{1} \theta_{d}\left(T_{f}\right)+A_{2} \theta_{d}\left(T_{f}-T_{2 A}\right)-B_{1} \theta_{d}\left(T_{f}\right)-B_{2} \theta_{d}\left(T_{f}-T_{2 B}\right), \\
u(t-T)=\left(\begin{array}{ll}
0 & \text { for } t<T \\
1 & \text { for } t>T
\end{array}\right)
\end{gathered}
$$

Fig.12 shows a simulation result using proposed input preshaping method. You can see that simple change of the reference input make clear response without vibration.

All calculation step is executed off-line, not a real-time estimation. If a reference trajectory is given, we can guess all parameters for input preshaping method from the frequency and joint configuration table. A thesis[4] proved that a proper IST filter with two impulses can eliminate a residual vibration of a time invariant system.

\section{EXPERIMENTAL RESULTS}

A PC-based UMAC motion controller[7] is used for controlling the six-dof safe arm, and MR dampers are independently controlled by a separated controller. Each encoder signal is sent to $\mathrm{PC}$ via USB and RS-232C port.
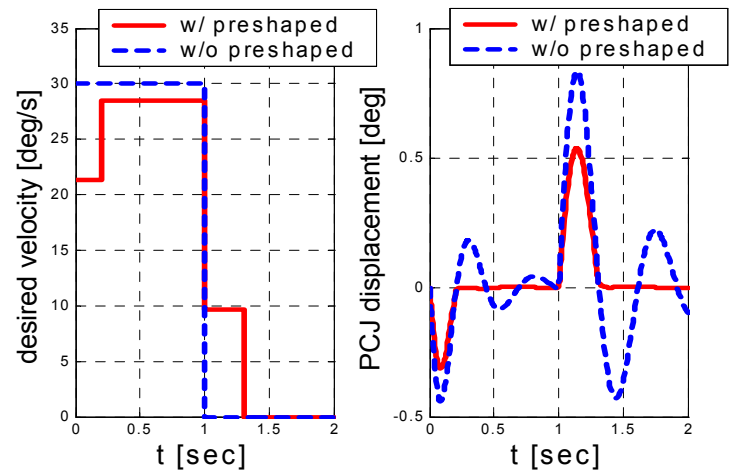

Fig. 12 Simulation: proposed input preshaping method

\section{A. With LTI system : 3rd joint control}

An experiment of $3 \mathrm{rd}$ joint of the safe arm is presented. A desired trajectory is $60 \mathrm{deg} / \mathrm{sec}$ during a second. Fig. 14 shows the result of the experiment. We can find that there is vibration of $3 \mathrm{~Hz}$ frequency. The implemented IST reduces vibration of 3rd axis dramatically. The difference between the PCJ displacement of a starting point and steady state is caused by gravity of the link. As shown in Fig.14(c), IST works well even in gravity. A little amount of overshoot shown in Fig.13(c) is still shown, but this is due to the inertia of the 3rd link not to the vibration mode. After the second impulse timing, little vibration is shown.
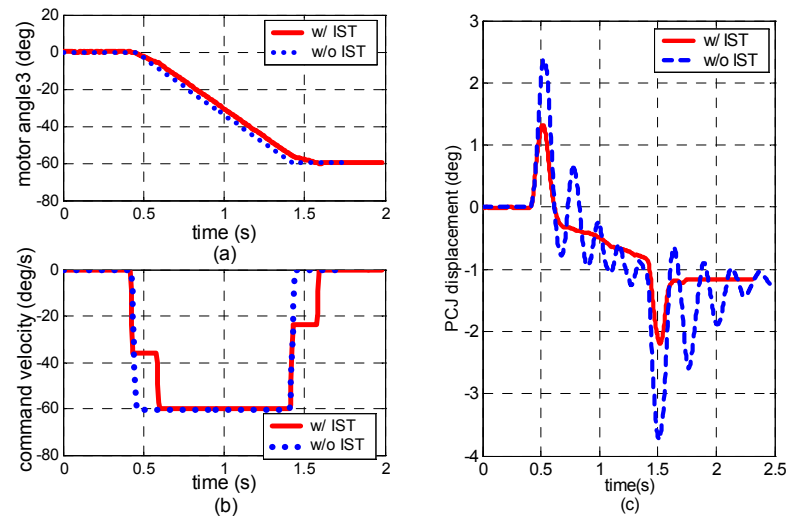

Fig.13 Experiment: vibration reduction for $3^{\text {rd }}$ axis:

(a) motor angle of the 3rd joint. (b) commandvelocity of the 3rd motor, (c) PCJ displacement of 3rd $\mathrm{PCJ}$ defined in equation(5)

\section{B. With LTV system : 2nd joint control}

To prove efficiency of the proposed algorithm, an experiment of 2 nd axis with varying inertia is performed. The joint angle of the 2 nd joint moves from 30 degree to zero degree, while the joint angle of the 3 nd joint rotates from -30 degree to +30 degree as shown in Fig.14. With a given trajectory and the table of period of vibration versus joint configuration, we can expect that the frequency of vibration varies from $1.85 \mathrm{~Hz}$ to $1.55 \mathrm{~Hz}$. Finally, we can generate the new trajectory by using proposed algorithm. Fig. 15 shows the preshaped input and the vibration of the 2nd PCJ. The controller by using the preshaped input clearly suppresses both 
of vibration including vibration of starting motion and ending motion.

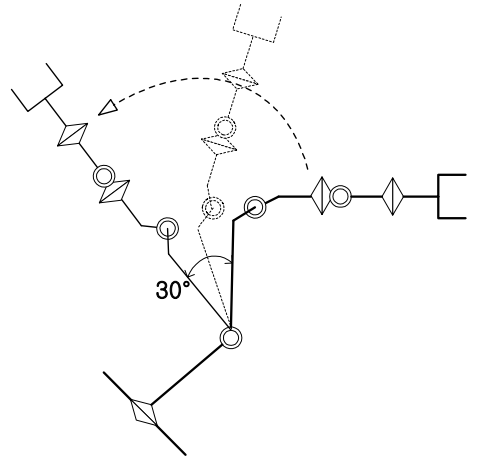

Fig. 14 change of joint configuration during the experiment

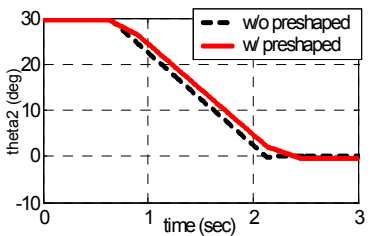

(a)

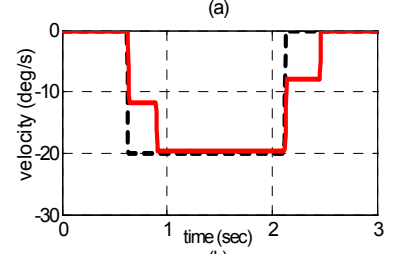

(b)

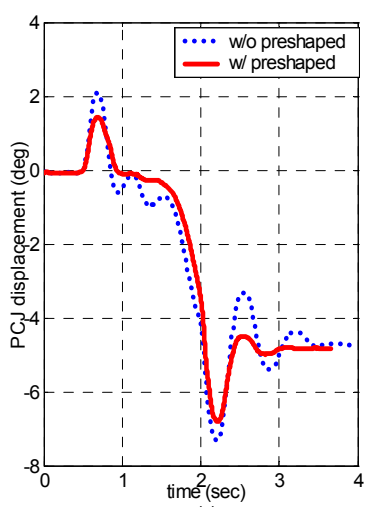

(c)

Fig. 15 Experiment: vibration reduction for 2nd axis: (a) motor angle of the 2nd joint, (b) command velocity of the 2nd motor, (c) displacement of the 2nd PCJ

\section{CONCLUSION}

The new safe arm for a service robot with passive compliant joints via springs and a MR damper and a covering is designed. A proper controller considering reduction of vibration by input-preshaping method is proposed for the safe arm. The vibration has two modes which are caused by starting motion and by ending motion respecrively. A reference input is shaped to reduce vibration. Parameters of impulse sequences by the modified TVIST and a conventional IST are used for input preshaping. Simulation results and experimental result shows that proposed method works properly on the safe arm.

\section{REFERENCES}

[1] S.-S. Yoon, S. Kang, S.-J. Kim, Y.-H. Kim, M. Kim, and C.-w. Lee," Safe Arm with MR-based Passive Compliant joints and Visco-elastic Covering for Service Robot Application," In Proceedings of the IEEE/RSJ International Conference on Intelligent Robots and Systems, USA, October 2003, pp. 2191-2196.

[2] M. Kim, S.-S. Yoon, S. Kang, S.-J. Kim, Y.-H. Kim, H.-S. Yim, C.-D. Lee, I.-T. Yeo, "Safe arm design for service robot," In Proceedings of The Second IARP -IEEE/RAS Joint Workshop on Technical Challenge for Dependable Robots in Human Environments, Toulouse, France, 2002, pp. 88-95.

[3] N.C. Singer, and W.P. Seering, "Preshaping Command Inputs to Reduce System Vibration," ASME Journal of Dynamic Systems, Measurement and Control, Vol.112, 1990, pp. 76-82.

[4] J. Park, P.H. Chang, and E. Lee, "Can a Time Invariant Input Shaping Technique Eliminate Residual Vibrations of LTV Systems," Proc. of the American Control Conference, 2002, pp. 2292-2297.

[5] B.W. Rappole, "Minimizing Residual Vibrations in Flexible Systems," Master thesis, Department of Mechanical Engineering, MIT, 1992.

[6] H.Y. Park, P.H. Chang, and J.S. Hur, "Time-Varying Input Shaping Technique Applied to Vibration Reduction of An Industrial Robot", Proc. of the IEEE/RSJ International Conference on Intelligent Robots and Systems, 1999, pp.285-290.

[7] The DeltaTau Corporation (http://www.deltatau.com/umacframe.htm) 